\title{
Le test de concordance de script comme outil d'enseignement et d'apprentissage : un projet-pilote pour les étudiants de première année de médecine
}

\section{The script concordance test as a teaching and learning tool: a pilot study for first-year medical students}

\author{
Léa HofF $^{1}$, Alain Bestawros ${ }^{1,2}$, Jeannine Kassis ${ }^{2}$ et Bernard Charlin ${ }^{1}$ \\ 1 Centre de pédagogie appliquée aux sciences de la santé (CPASS), Faculté de médecine, Université de Montréal, \\ Canada \\ 2 Programme d'hématologie, Département de médecine, Faculté de médecine, Université de Montréal, Canada
}

Manuscrit reçu le 24 août 2009 ; commentaires éditoriaux formulés aux auteurs le 25 avril 2010 et le 9 mai 2010 ; accepté pour publication le 9 mai 2010

Mots clés :

Éducation médicale ; test de concordance de script ; activité éducative ; raisonnement clinique

\section{Keywords:}

Medical education; script concordance test; educational activity; clinical reasoning
Résumé - Contexte : Les étudiants en médecine peuvent éprouver de la difficulté à organiser leurs connaissances théoriques d'une façon qui soit adaptée à la pratique clinique. Méthodes : Le test de concordance de script (TCS), outil habituellement utilisé pour évaluer le raisonnement clinique, a été employé dans un but pédagogique pour amener les étudiants à utiliser en contexte clinique les connaissances qu'ils viennent d'acquérir. L'activité de petit groupe a été réalisée pour les étudiants de première année à la fin du cours de Sciences Hématologiques à l'Université de Montréal. Un questionnaire anonyme demandant aux étudiants de décrire les points forts et les points faibles de l'activité a été administré à la fin de l'activité. Résultats : L'activité a été appréciée à la fois par les étudiants et par les tuteurs. Les points forts identifiés par les étudiants incluent l'occasion de discuter et réviser les notions apprises, d'intégrer leurs connaissances et de développer le raisonnement clinique. Conclusion : Le TCS peut être employé de façon formative pour des étudiants n'ayant que peu d'expérience clinique. Les réflexions et discussions induites par le format des questions semblent favoriser l'intégration des connaissances.

Abstract - Context: Medical students may have difficulty organizing basic-science knowledge in a way that is clinically relevant. Methods: The Script Concordance Test (SCT), which is a tool generally used to assess clinical reasoning, was used in an educational purpose to help students apply recently acquired knowledge in a clinical context. A small-group activity was offered to first-year students at the end of the Hematological Sciences course at the University of Montreal. At the end of the activity, students took a survey asking them to highlight the activity's strengths and weaknesses. Results: Students and tutors alike found the activity worthwhile. Strengths identified by students included the opportunity to debate and review 
the concepts learned, the integration of knowledge and the development of clinical reasoning skills. Conclusion: The SCT can be used as a learning tool for students with little clinical experience. The reflective thinking and discussions brought on by the question format seem to facilitate the knowledge integration.

\section{Introduction}

L'objectif de tout programme de médecine est de former des médecins compétents qui possèdent non seulement de solides connaissances scientifiques mais qui sont également capables de mobiliser ces connaissances efficacement dans leur pratique, en démontrant un bon jugement clinique. Le raisonnement clinique est l'activité intellectuelle qui sélectionne les informations obtenues à partir d'une situation clinique spécifique, qui l'intègre aux connaissances et expériences antérieures et l'utilise pour prendre des décisions de diagnostic et de prise en charge du patient ${ }^{[1]}$.

À l'Université de Montréal et ailleurs, plusieurs stratégies ont été mises en exergue pour initier les étudiants à la réalité de la pratique clinique dès le début de leur formation. Nous rapportons ici l'utilisation d'un test de concordance de script (TCS) comme outil éducatif afin d'aider les étudiants de première année de médecine à organiser leurs connaissances dans une optique davantage centrée sur l'exercice clinique.

Le test de concordance de script (TCS) est un outil qui a été développé afin d'évaluer le raisonnement clinique, à travers l'interprétation des données cliniques en contexte d'incertitude. Les cas cliniques d'un TCS comportent une dimension d'ambiguïté, reflétant ainsi la complexité des situations cliniques réelles ${ }^{[2]}$. Les problèmes cliniques complexes (illstructured) se caractérisent par des données manquantes, l'absence d'une solution standardisée (il existe plusieurs solutions raisonnables) ou un désaccord des experts quant à la meilleure solution ${ }^{[3]}$. La tâche des étudiants consiste à interpréter la compatibilité de nouvelles données présentées avec l'une des hypothèses pertinentes par rapport à la situation. Les réponses de l'étudiant sont recueillies à l'aide d'une échelle de Likert et l'outil mesure leur degré de concordance avec celles formulées par un panel d'experts ${ }^{[4]}$.

Le TCS est habituellement utilisé pour évaluer des étudiants, des résidents et des médecins diplômés. Le but de cette étude était d'évaluer la possibilité d'utiliser cet outil à des fins d'apprentissage ${ }^{[5,6]}$, pour faciliter l'intégration des connaissances, et ce chez des étudiants n'ayant que peu d'expérience clinique.

\section{Collectifs et méthodes}

L'étude a été menée auprès de 264 étudiants de première année de médecine à l'Université de Montréal, au terme d'une séquence de sessions d'apprentissage par problème (APP) en sciences hématologiques. Une activité éducative d'une durée d'environ 90 minutes, basée sur le format du TCS a été organisée à la fin du cours. Pour cette activité, les étudiants étaient divisés en groupes de huit, répartis dans différentes salles et accompagnés du médecin qui était leur tuteur pour ce cours.

Chaque cas du TCS débute par une vignette clinique inspirée de la pratique quotidienne de l'hématologie. Une hypothèse diagnostique est ensuite proposée. La tâche de l'étudiant consiste à évaluer l'impact d'une nouvelle donnée clinique sur le diagnostic proposé. Les questions ont été construites selon le modèle classique du TCS, en trois parties ${ }^{[4]}$ (cf. exemples sur le tableau I). Initialement, une banque de 13 cas cliniques et 50 questions a été conçue par une ex-étudiante du cours (LH) puis révisée par deux hématologues (JK et $\mathrm{AB}$ ) pour vérifier que les questions reflétaient les objectifs pédagogiques du cours et la réalité clinique. Afin de créer une grille de réponse d'experts, il a été demandé aux tuteurs du cours de compléter le TCS en ligne dans un délai de deux semaines. Parmi 
Tableau I. Exemples de cas du test de concordance de script en hématologie.

Cas 1: Un homme de 23 ans vous consulte pour fatigue et parce qu'il a remarqué des bosses dans son cou depuis quelques jours. À l'examen, vous trouvez une adénopathie cervicale bilatérale et le signe de percussion splénique est positif.

\begin{tabular}{|l|l|c|c|c|c|c|}
\hline Si vous pensiez à : & Et que vous trouvez : & \multicolumn{3}{|c|}{ Cette hypothèse devient : } \\
\hline $\begin{array}{l}\text { Une mononucléose } \\
\text { infectieuse }\end{array}$ & Des adénopathies cervicales douloureuses & -2 & -1 & 0 & +1 & +2 \\
\hline $\begin{array}{l}\text { Un lymphome non Hodgkin } \\
\text { Un lymphome de Hodgkin }\end{array}$ & $\begin{array}{l}\text { Le patient est porteur du VIH } \\
\text { Le patient rapporte une sensation douloureuse } \\
\text { rétrosternale après avoir bu de l'alcool }\end{array}$ & -2 & -1 & 0 & +1 & +2 \\
& -1 & 0 & +1 & +2 \\
\hline
\end{tabular}

Cas 2 : Un homme de 57 ans vous consulte parce qu'il se sent fatigué et fiévreux depuis quelques jours. II a également remarqué que ses gencives saignent lorsqu'il se brosse les dents. À l'examen, vous notez une pâleur des conjonctives et une température de $38,5^{\circ} \mathrm{C}$.

\begin{tabular}{|l|l|c|c|c|c|c|}
\hline Si vous pensiez à : & Et que vous trouvez : & \multicolumn{3}{|c|}{ Cette hypothèse devient : } \\
\hline Une leucémie myéloïde aiguë & $\begin{array}{l}\text { Le patient a reçu de la chimiothérapie il y a 10 ans } \\
\text { pour un lymphome de Hodgkin }\end{array}$ & -2 & -1 & 0 & +1 & +2 \\
\hline $\begin{array}{l}\text { Un syndrome myélodyspla- } \\
\text { sique }\end{array}$ & $\begin{array}{l}\text { La NFSC faite il y a un an montre une légère bi- } \\
\text { cytopénie dont une anémie macrocytaire pour la- } \\
\text { quelle il reçoit de la vitamine B12 }\end{array}$ & -2 & -1 & 0 & +1 & +2 \\
\hline
\end{tabular}

$-2=$ improbable $;-1=$ moins probable $; 0=$ ça ne change rien $;+1=$ plus probable $;+2=$ très probable.

VIH : virus de l'immunodéficience humaine.

NFSC : numération formule sanguine complète.

les 32 tuteurs éligibles, 20 tuteurs ont répondu à toutes les questions. Ce nombre dépasse le nombre minimal de 15 panélistes, jugé nécessaire pour atteindre une bonne fiabilité statistique ${ }^{[7]}$. Les tuteurs n'étaient pas tous hématologues mais tous maîtrisaient bien le contenu du cours. Dix étaient hématologues ou hémato-oncologues, trois étaient radiooncologues, deux étaient médecins de famille, un était interniste, un était microbiologiste et trois n'ont pas précisé leur spécialité. Le test a été optimisé à partir des réponses données par les membres du panel ${ }^{[8]}$. Les questions ayant suscité une grande variabilité parmi les réponses des experts ont été éliminées. Certains cas explorant la même section de la matière ont également été retirés pour tenir compte de la durée limitée de l'activité. Finalement, le TCS soumis aux étudiants comprenait sept cas cliniques et 16 questions, qui portaient sur des problèmes fréquents en hématologie.

Lors de l'activité d'apprentissage, les cas et les questions étaient affichés sur l'écran de l'ordinateur de chacune des salles de rencontre. Dans chaque salle, les étudiants discutaient et répondaient aux questions du TCS en groupes de huit. Pour chaque question, les étudiants évaluaient ensemble l'impact de nouvelles données cliniques sur la probabilité d'une hypothèse diagnostique qui leur était proposée. Puisqu'ils ne pouvaient fournir qu'une seule réponse par salle, les étudiants devaient débattre des questions et justifier leur raisonnement à leurs collègues. Ils entraient ensuite la réponse consensuelle du groupe dans l'ordinateur. Bien que l'activité soit formative, la réponse du groupe était affectée d'un score pour chaque question, calculé à partir des réponses du panel d'experts. Les médecins tuteurs du cours étaient présents avec leur groupe pour animer la discussion et suggérer des pistes de réflexion. Après que les étudiants aient répondu à une question, le tuteur présentait la distribution des réponses fournies par le panel d'experts et donnait des explications cliniques au besoin. À la fin de l'activité, les étudiants ont répondu à un questionnaire 
anonyme. Le questionnaire comprenait huit questions sur échelle de Likert concernant l'appréciation de l'activité et quatre questions ouvertes (points forts, points faibles, suggestions pour l'amélioration et autres commentaires). En accord avec certains commentaires méthodologiques récents, les variables ordinales générées par les réponses sur les échelles de Likert ont été traitées comme des variables à intervalles ${ }^{[9]}$.

\section{Résultats}

Tous les groupes d'étudiants ont répondu aux 16 questions du TCS et la majorité a complété le test en moins de 60 minutes. Les réponses au questionnaire anonyme ont montré que les étudiants ont apprécié l'activité. Parmi les 264 étudiants, 195 ont répondu aux questions sur échelle de Likert (taux de réponse 73,86\%) (figure 1) et 102 étudiants ont répondu à au moins une question ouverte (taux de réponse 38,64\%). Les points forts identifiés par les étudiants dans les questions ouvertes incluent l'occasion de discuter et réviser les notions apprises $(54,90 \%$ des répondants), d'intégrer leurs connaissances $(12,75 \%)$ et de développer le raisonnement clinique $(10,78 \%)$. Ils commentent : «cette activité permet de discuter et de consolider ses connaissances »; "force à réfléchir et faire des liens, se rapproche de la pratique » et «permet de voir que la réalité médicale n'est ni blanche ni noire ». Les points faibles signalés incluent la difficulté de répondre aux questions sur l'échelle de Likert (format d'interrogation nouveau pour les étudiants) (10,78\% des répondants) et l'ambiguïté de certaines questions $(9,80 \%)$. Les suggestions incluent l'ajout d'un plus grand nombre de questions $(13,73 \%$ des répondants) et la mise à disposition de l'activité sur Internet $(8,82 \%)$. Les tuteurs du cours ont également apprécié l'activité. Leurs commentaires ont été récoltés de manière informelle par le responsable du cours. Les tuteurs ont indiqué que l'activité favorise chez les étudiants l'assimilation du savoir et la production de diagnostics différentiels.

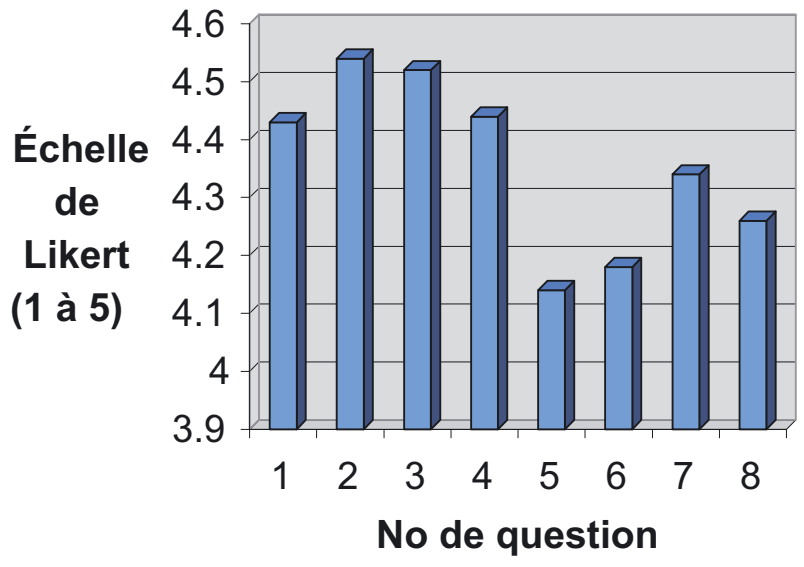

Fig. 1. Niveau de satisfaction des étudiants suite à l'activité test de concordance de script.

Questions : 1 : L'activité d'apprentissage était appropriée à mon niveau de formation (Moy 4,43, DS 0,65); 2 : Le contexte en groupes avec tuteurs était propice pour cette activité (Moy 4,54, DS 0,71) ; 3 : La durée de la séance était suffisante (Moy 4,52, DS 0,73); 4 : Le moment était bien choisi pour cette activité (Moy 4,44, DS 0,97); 5 : Cette activité m'a permis de réviser les concepts importants appris dans ce cours (Moy 4,14, DS 0,92); 6 : Cette activité était utile à ma formation (Moy 4,18, DS 0,85 ) ; 7 : Cette activité était agréable (Moy 4,43, DS 0,84) ; 8 : J'aimerais refaire cette activité dans d'autres cours (Moy 4,26, DS 0,96). Moy : moyenne, DS : déviation standard.

Échelle de Likert à 5 ancrages (1: Totalement en désaccord à 5 : Totalement d'accord). Bien qu'il existe un débat dans la littérature sur la légitimité de cette façon de procéder ${ }^{[9]}$, les scores sont issus du calcul de moyennes des réponses obtenues sur l'échelle de Likert.

\section{Discussion}

Traditionnellement, en Amérique du Nord, les étudiants en médecine acquièrent des connaissances des sciences fondamentales durant les deux premières années de leur formation et reçoivent une formation pratique durant les deux dernières années (externat à temps plein). Les activités éducatives visant à développer le raisonnement clinique sont généralement offertes en fin de cursus, grâce à la supervision au cours des stages cliniques ou par des activités formelles spécifiques telles que les séances $\mathrm{d}$ apprentissage du raisonnement clinique ${ }^{[10]}$ et les discussions de cas cliniques. Le savoir théorique appris au début de la formation doit donc être réorganisé pour être utilisé de façon efficace en clinique ${ }^{[6]}$. 
Cette transition n'est pas toujours facile et il serait avantageux d'aider les étudiants à reconnaître très tôt les applications cliniques de leurs savoirs théoriques.

Le travail de Williams et al. ${ }^{[6]}$ montre que les étudiants en médecine acquièrent progressivement le raisonnement clinique et ce, dès la première année d'études médicales. Des activités visant à initier les étudiants au raisonnement clinique et à stimuler la construction des «scripts cliniques » (connaissances spécifiquement organisées pour des actions cliniques diagnostiques ou thérapeutiques ${ }^{[11]}$ ) peuvent donc être offertes dès le début de leur formation. Par exemple, Custers et al. ${ }^{[12]}$ proposent une méthode d'analyse de problèmes cliniques qui systématise l'enseignement de la résolution de problèmes cliniques et qui peut être utilisée dès le début des études médicales.

Jusqu'à présent, le TCS a surtout été utilisé comme instrument d'évaluation du raisonnement clinique des résidents et des médecins. Petrella ${ }^{[5]}$ rapporte l'utilisation du TCS comme outil d'apprentissage en formation médicale continue. L'expérience décrite ici montre que cet outil peut également être utilisé dans un but pédagogique avec des étudiants n'ayant pas d'expérience clinique. L'utilisation du TCS comme outil de formation pour des étudiants novices pourrait permettre de stimuler explicitement l'acquisition de scripts cliniques en favorisant la verbalisation des connaissances et du processus de raisonnement. De plus, le TCS est utile pour permettre l'intégration des notions apprises et pour initier les étudiants à la difficulté d'interpréter des données cliniques dans les situations mal définies qui caractérisent souvent la pratique clinique.

L'expérience décrite a donné des résultats prometteurs, mais la portée limitée de l'activité ne permet pas d'évaluer pour l'instant les impacts qu'une telle innovation pourrait avoir sur la consolidation des connaissances ou l'acquisition du raisonnement clinique par les étudiants. L'activité a été jugée utile et intéressante par les étudiants et les tuteurs. Ils recommandent son utilisation dans d'autres cours. Pour donner suite à ces données très positives, l'activité sera poursuivie et approfondie l'an prochain au sein du cours d'hématologie et il sera suggéré aux responsables des autres cours d'en tester l'utilisation dans leur discipline.

\section{Contributions}

Léa Hoff a conçu l'activité d'apprentissage, rédigé le matériel à destination des étudiants, organisé l'activité, recueilli et analysé les données et rédigé le manuscrit. Alain Bestawros a conçu l'activité d'apprentissage, rédigé le matériel à destination des étudiants, organisé l'activité et révisé le manuscrit. Jeannine Kassis a révisé le matériel à destination des étudiants, organisé l'activité et révisé le manuscrit. Bernard Charlin a conçu l'activité d'apprentissage et contribué à la rédaction du manuscrit.

\section{Remerciements}

Les auteurs tiennent à remercier Driss Kazitani pour la mise en ligne du TCS.

Ce travail a fait l'objet d'une bourse de recherche en éducation octroyée par la faculté de médecine de l'Université de Montréal (bourse COPSECPASS).

\section{Références}

1. Charlin B, Bordage $\mathrm{G}$, van der Vleuten. L'évaluation du raisonement clinique. Pédagogie Médicale 2003;4:4252.

2. Charlin B, Gagnon R, Lubarsky S, Lambert C, Meterissian $\mathrm{S}$, Chalk $\mathrm{C}$ et al. Assessment in the context of uncertainty using the script concordance test: more meaning for scores. Teach Learn Med 2010;22:180-8.

3. Nguyen DQ, Blais JG. Approche par objectifs ou approche par compétences ? Repères conceptuels et implications pour les activités d'enseignement, d'apprentissage et d'évaluation au cours de la formation clinique. Pédagogie Médicale 2007;8:232-51.

4. Carriere B, Gagnon R, Charlin B, Downing S, Bordage G. Assessing clinical reasoning in pediatric emergency medicine: validity evidence for a Script Concordance Test. Ann Emerg Med 2009;53:647-52. 
5. Petrella RJ, Davis P. Improving management of musculoskeletal disorders in primary care: the Joint Adventures Program. Clin Rheumatol 2007;26:1061-6.

6. Williams RG, Klamen DL, Hoffman RM. Medical student acquisition of clinical working knowledge. Teach Learn Med 2008;20:5-10.

7. Gagnon R, Charlin B, Coletti M, Sauve E, van der Vleuten C. Assessment in the context of uncertainty: how many members are needed on the panel of reference of a script concordance test. Med Educ 2005;39:284-91.

8. Fournier JP, Demeester A, Charlin B. Script Concordance Tests: Guidelines for construction. BMC Med Inf Decis Mak 2008;8:18.

9. Carifio J, Perla R. Resolving the 50-year debate around using and misusing Likert scales. Med Educ 2008;42:1150-2.
10. Chamberland M. Les séances d'apprentissage du raisonnement clinique (ARC) : Un exemple d'activité pédagogique contextualisée adaptée aux stages cliniques en médecine. Ann Med Interne 1998;149:479-484

11. Nendaz M, Charlin B, Leblanc V, Bordage G. Le raisonnement clinique: données issues de la recherche et implications pour l'enseignement. Pédagogie Médicale 2005;6:235-54.

12. Custers EJ, Stuyt PM, De Vries Robbe PF. Clinical problem analysis (CPA): a systematic approach to teaching complex medical problem solving. Acad Med 2000;75:291-7.

Correspondance et offprints : Léa Hoff, Université de Montréal, CPASS-Recherche, pièce S-312, 2900, Bd. Édouard-Montpetit, Pavillon Roger-Gaudry, Montréal, Québec H3T 1J4 Canada.

Mailto : lea.hoff.arcand@umontreal.ca 